\title{
A Correlation-Maximization Denoising Filter Used as An Enhancement Frontend for Noise Robust Bird Call Classification
}

\section{Wei Chu and Abeer Alwan}

Speech Processing and Auditory Perception Laboratory

Department of Electrical Engineering University of California, Los Angeles

Supported in part by the NSF 


\section{Outline}

- Motivation

- Bird Call Analysis

- Bird Call Classifier Design

- Denoising Filter Design

- Experiments 


\section{Motivation of noise robust bird call classification}

- Songs are important in the communication between birds of specific species.

- Behavioral and ecological studies could benefit from automatically detecting and identifying species from acoustic recordings.

- It is a challenge to correctly classify the bird calls under noisy conditions.

- In this work, we analyze 5 types of Antbirds.

Now let us listen to several examples of Antbird calls: 


\section{Waveform and spectrogram of a Barred Antshrike (BAS) call}

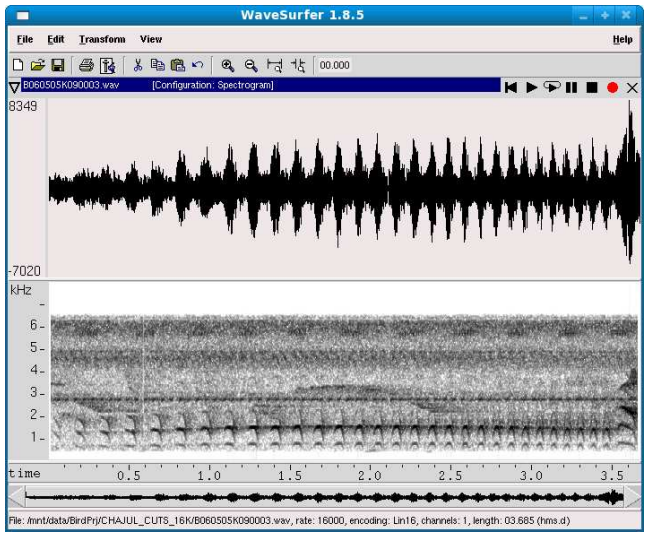




\section{Waveform and spectrogram of a Dusky Antbird (DAB) call}

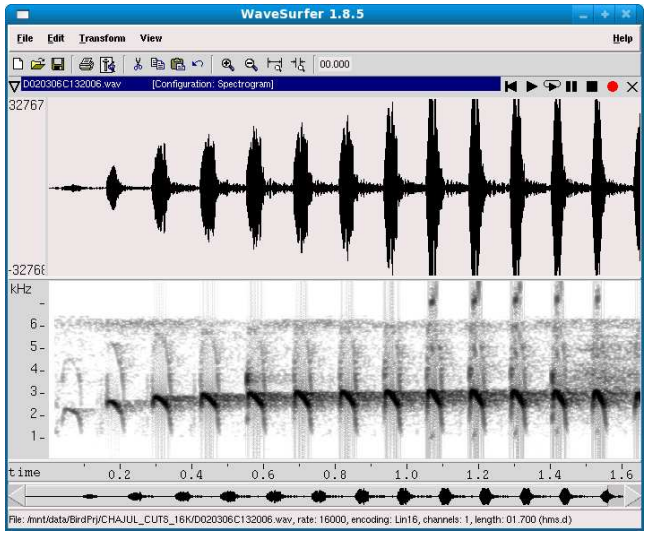




\section{Waveform and spectrogram of a Great Antshrike (GAS) call}

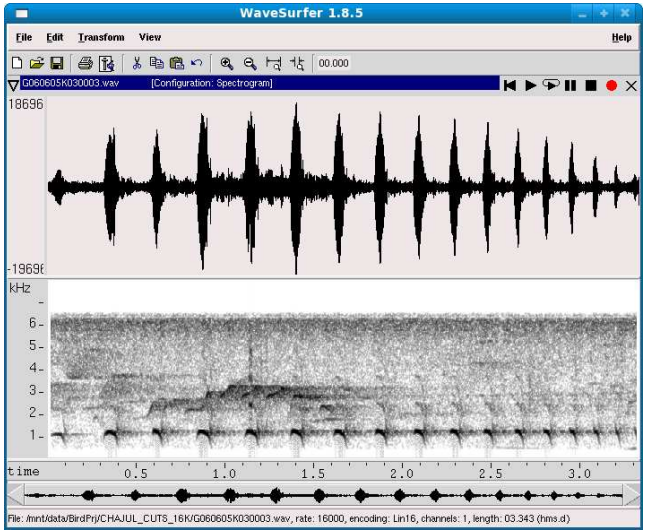




\section{Waveform and spectrogram of a Mexican Antthrush (MAT) call}

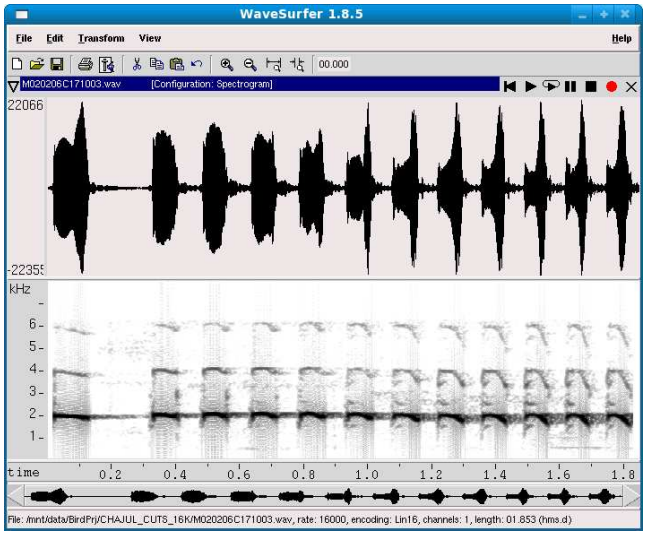




\section{Waveform and spectrogram of a Dot-winged Antwren (DWA) call}

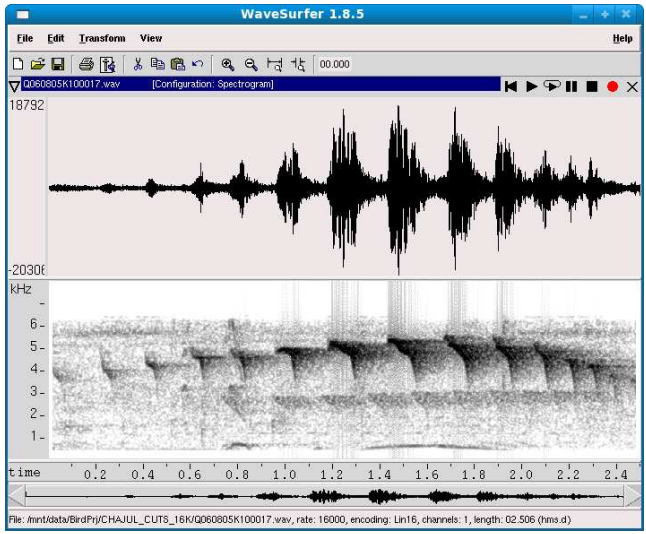




\section{Antbird Call Properties}

- A bird call consists of a sequence of chirps.

- The interval between chirps and the chirp intensity gradually decrease over time.

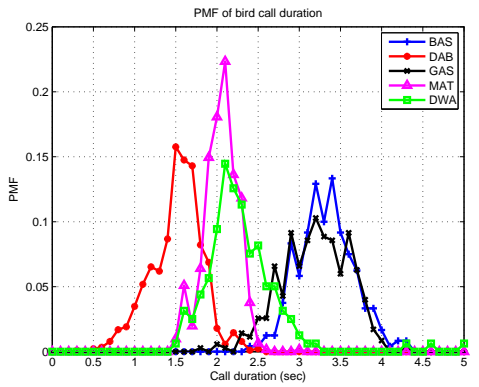

A histogram of bird call duration of 2246 samples from 5 bird species. The duration ranges from 0.5 to 5 seconds. 


\section{Automatic bird call classification involves several aspects:}

- Waveform denoising: the focus of this paper

- Feature extraction: Mel-Frequency Cepstral Coefficients (MFCCs)

- Acoustic modelling: Gaussian Mixture Model (GMM) and Hidden Markov Model (HMM)

- Learning model parameters from observations

- Decoding observations 


\section{Why denoising is needed?}

Different kinds of background noise can be observed in the recordings:

- Other bird chirps

- Insect sounds

- Sounds of other animals

We propose a Correlation-Maximization based filter to suppress background noise existed in the bird calls. 


\section{Wiener Filter}

\section{A prevailing denoising approach: Wiener filtering}

Clean $X(f)$ is corrupted by an additive noise $\Rightarrow$ noisy $Y(f)$. $\operatorname{SNR}(f)$ : an estimation of $\operatorname{SNR}(f)$ :

$$
\operatorname{SNR}(f)=\frac{|\hat{X}(f)|^{2}}{|\hat{N}(f)|^{2}}
$$

The estimated clean spectrum is :

$$
|\hat{X}(f)|^{2}=H(f)|Y(f)|^{2}=\frac{\mathrm{S} \widehat{\mathrm{NR}}(f)}{1+\mathrm{SNR}(f)}|Y(f)|^{2}
$$

The noncausal Wiener filter converts the denoising problem into an SNR estimation problem [1]. 


\section{Correlation-Maximization Filter}

\section{Futher Analysis of the Bird Call}

\section{Two Levels of Bird Call Periodicity}

(1) Short phonation period (Left): ranges from $0.2-1.0 \mathrm{~ms}$

(2) Interval between chirps (Right): ranges from $0.06-0.3 \mathrm{sec}$, slowly decreases with time. $\Rightarrow$ instruct the denoising!
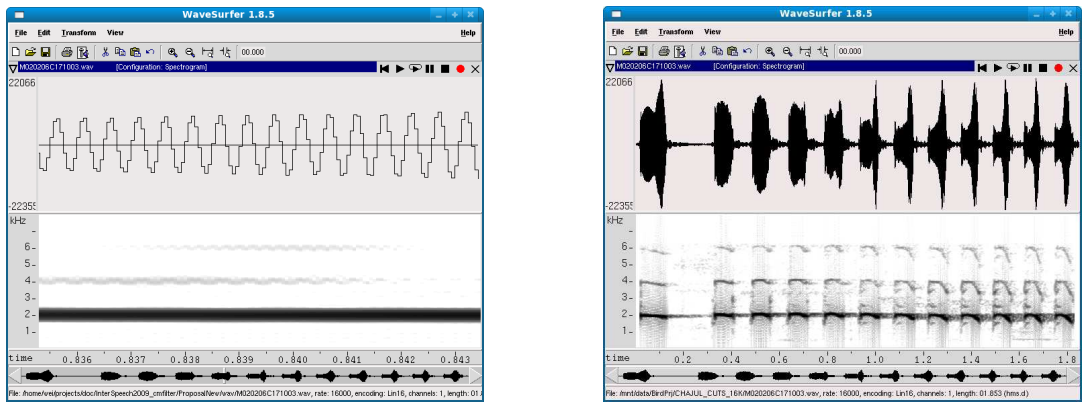


\section{Correlation-Maximization Filter}

\section{Correlation-Maximization Filter}

Suppose an FIR filter with $L$ taps:

$$
\mathbf{h}=[h[1], h[2], \cdots, h[L]]^{T}
$$

is used for denoising the noisy bird call $y[n]$.

The output of the filter is the estimated clean signal $\hat{x}[n]$ :

$$
\hat{x}[n]=\sum_{k=1}^{L} h[k] y[n-k]
$$

$y[n]$ and $\hat{x}[n]$ is then segmented into frames. 


\section{Correlation-Maximization Filter}

\section{Correlation-Maximization Filter (cont.)}

\section{Two Assumptions}

(1) $y[n]$ and $\hat{x}[n]$ are wide sense stationary: The bird chirps are repeating periodically.

(2) A single $\mathbf{h}$ for each bird call: The spectral distributions of different frames in a bird call are similar.

The cross correlation function of $\hat{x}[n]$ at lag $k$ of frame $m$ :

$$
\phi_{\hat{x}}^{m}[0, k]=\mathbf{h}^{T} \Phi_{y}^{m}[0, k] \mathbf{h}
$$

$\mathbf{h}=[h[0], h[1], \cdots, h[L]]^{T}:$ coefficients of the FIR filter. $\Phi_{y}^{m}[0, k]$ : cross correlation function of $y[n]$ (independent of $\mathbf{h}$ ) 


\section{Correlation-Maximization Filter}

\section{Use Dynamic Programming (DP) to Search the Chirp Interval}

Searching the chirp interval in each frame over $\hat{x}[n]$.

DP: minimizing the distortion induced by background noise

- Local cost at lag $k$ of frame $m:-\bar{\phi}_{\hat{x}}^{m}[0, k]$

- Transition cost of from lag $k_{i}$ at to $k_{j}$ :

$$
d\left(k_{i}, k_{j}\right)=e^{\alpha\left|k_{i}-\delta-k_{j}\right|}-1
$$

Purpose: prevent chirp intervals from greatly varying in two consecutive frames.

A trellis structure of $K \times M$ for dynamic programming is built. 


\section{Correlation-Maximization Filter}

\section{Correlation-Maximization Filter (cont.)}

The effect of an optimal filter $\mathbf{h}$

Removing the additive noise in the corrupted signal so that the minimum accumulative cost is achieved in chirp interval searching:

$$
\mathbf{h}^{*}=\underset{\mathbf{h}}{\arg \min } \mathcal{F}(\mathbf{h}, \mathbf{s})
$$

s: an valid path in the trellis: $\mathbf{s}=s_{1}, s_{2}, \cdots, s_{M}$, $\mathbf{h}^{*}$ : the optimal denoising filter. the accumulative cost $\mathcal{F}(\mathbf{h}, \mathbf{s})=\Psi(\mathbf{h}, \mathbf{s})+\Theta(\mathbf{h}, \mathbf{s})$. $\Psi(\mathbf{h}, \mathbf{s})$ : accumulative local cost; $\Theta(\mathbf{h}, \mathbf{s})$ : accumulative transition cost. 


\section{Speed Up: From Brute Force to N-Best}

- There are $K^{M}$ possible paths in a $K \times M$ trellis. Suppose the average iteration times of the gradient search is $\bar{l}$, this brute-force approach needs $K^{M} \times \bar{l}$ iterations which is computationally unacceptable.

- We can assume that $\mathbf{s}^{*}$ is within a path subset denoted by $\mathcal{S}(\mathbf{h})$ in each iteration. The subset is composed of the top $\mathrm{N}$-best paths from the dynamic programming using the trellis.

- That means the gradient descent search is only needed to be applied to the $\mathrm{N}$-best paths, not all the paths at each iteration.

- Let $J$ denotes the size of $\mathrm{N}$-best search, the total gradient search iterations is reduced to $J^{2} \times \bar{T}$.

- Typically, for Antbird calls, $K=49,1 \leq M \leq 50, J=20$. 


\section{The spectrograms of a GAS call before and after filtering}

- (a) other non-target bird chirps: 0.6 - 1.6 seconds

- (b) both target and non-target bird chirps are enhanced after Wiener filtering

- (c) Correlation-Maximization filter suppressed the non-target chirps while enhancing the target chirps

- (d) non-target chirps and background noise are suppressed when cascading two filters

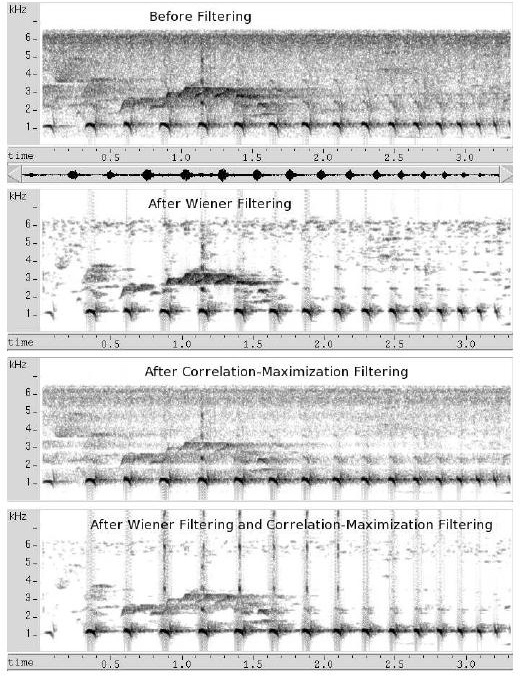




\section{Correlation-Maximization Filter}

\section{The frequency response of the CM filter for a GAS call}

- enhanced the the target bird call;

- minimized the interference introduced by background noise and other bird.

\section{filter h's characteristic}

- pass-band: $800-1750 \mathrm{~Hz}$

- stop-band: $2600-8000 \mathrm{~Hz}$

- dip: around $2800 \mathrm{~Hz}$

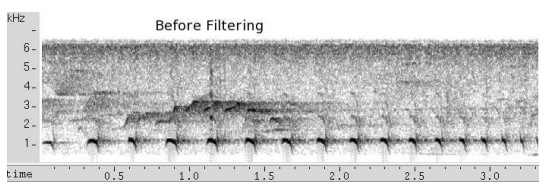

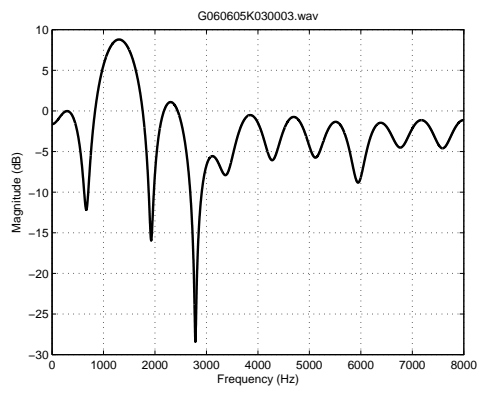




\section{Data Set}

Researchers from UCLA Ecology and Evolutionary Biology department collected 2 hours of bird calls (3366 calls) from 5 species. We split the corpus into a training and testing set with a ratio of $2: 1$.

Table: 2.1 The number of bird calls in the training and test sets. BAS: Barred Antshrike; DAB: Dusky Antbird; GAS: Great Antshrike; MAT: Mexican Antthrush; DWA: Dot-winged Antwren.

\begin{tabular}{|l||c|c|c|c|c||c|}
\hline & BAS & DAB & GAS & MAT & DWA & Total \\
\hline \hline Training & 240 & 888 & 350 & 609 & 159 & 2246 \\
\hline Testing & 120 & 444 & 175 & 304 & 77 & 1120 \\
\hline
\end{tabular}

The training set has 85 minutes of recordings; the testing set is 42 minutes long. 


\section{Setting}

- A band-pass filter with cutoff frequencies at $360 \mathrm{~Hz}$ and $6500 \mathrm{~Hz}$ is used to remove the irrelevant frequency components.

- Downsamped from $44.1 \mathrm{kHz}$ to $16 \mathrm{kHz}$.

- The taps of the filter $L=20$.

- The frame length $N=600 \mathrm{~ms}=9600$ samples.

- The dimensions of MFCC features is 39.

- GMM: 256 Gaussians; HMM: 6 states, 256 Gaussians / state. 


\section{Classification Results Analysis}

Table: 2.2 The classification error rate using the bird call test set. $\mathbf{W}_{+} / \mathbf{C M}_{\mathbf{+}}$ : feature extraction using the output of the Wiener/Correlation-Maximization based denoising filter

\begin{tabular}{|l||c|c|}
\hline & GMM & HMM \\
\hline \hline MFCC & $8.7 \%$ & $5.4 \%$ \\
\hline W+MFCC & $5.9 \%$ & $4.9 \%$ \\
\hline CM+MFCC & $5.3 \%$ & $4.6 \%$ \\
\hline CM+W+MFCC & $\mathbf{4 . 7} \%$ & $\mathbf{4 . 1} \%$ \\
\hline
\end{tabular}

- HMM based classifier is better than the GMM classifier when using the same features.

- Correlation-Maximization based denoising filter is effective before extracting MFCC features.

- Cascading the CM filter and Wiener filter is most effective. 


\section{Conclusions and Future Work}

The Correlation-Maximization based denoising filter is effective in reducing the classification errors of the bird call which has a quasi-periodic structure in the time domain and an invariant power spectral density across frames.

\section{Future work}

- Extract better features for classification, such as long-term features and the modulation frequency features;

- Detect the bird call in an audio stream.

- Use Dynamic Bayesian Network to represent the probabilistic relationships between the observed bird calls and the bird species. 


\section{Thank you!}

$Q \& A$ ? 
S. Boll, "Suppression of acoustic noise in speech using spectral subtraction,"

IEEE Trans. on Acoustics, Speech and Signal Processing, vol. 27, no. 2, pp. 113-120, 1979. 